\title{
Polysaccharide extracted from WuGuChong reduces high-fat diet-induced obesity in mice by regulating the composition of intestinal microbiota
}

Wendong Wang ${ }^{1,2+}$, Mintao Zhong ${ }^{3+}$, Tiantian Yu ${ }^{4}$, Lei Chen ${ }^{3}$, Lijun Shi ${ }^{1}$, Junwei Zong ${ }^{1 *}$ and Shouyu Wang ${ }^{1,2^{*}}$

\begin{abstract}
Background: Obesity is a severe public health threat worldwide. Emerging evidence suggests that gut microbiota dysbiosis is closely associated with obesity and its related metabolic complications. The aim of the present study is to investigate the effects of polysaccharide extracted from WuGuChong (PEW) on high-fat diet (HFD)-induced obesity, and the potential mechanisms involving modulation of the gut microbiota composition.

Methods: Mice were fed a normal chow diet and a high-fat diet with or without PEW ( $300 \mathrm{mg} / \mathrm{kg} /$ day) by oral gavage for 8 weeks. Body weight, obesity-related metabolic disorders, and gut microbiota were examined at the end of the experiment.

Results: PEW supplementation reduces body weight, adipose hypertrophy, liver steatosis, insulin resistance and systemic inflammation in HFD-fed mice, as well as maintains intestinal epithelium integrity. High-throughput $16 \mathrm{~S}$ rRNA analysis demonstrates that PEW supplementation alters the composition of gut microbiota. The Firmicutes to Bacteroidetes ratio and the relative abundance of Proteobacteria are increased in HFD-fed mice, which are reversed by PEW supplementation to approximately the control levels.
\end{abstract}

Conclusions: Our results suggest that PEW may be used as a bioactive ingredient to prevent obesity and its related metabolic disorders by modulating the composition of gut microbiota.

Keywords: Gut microbiota, High-fat diet, Obesity, Polysaccharides, WuGuChong

\section{Background}

Obesity is a global public health issue, and the major causes of obesity are considered to be unhealthy dietary patterns and lifestyles [1,2]. Accumulating evidence suggests that obesity, which is closely related to chronic systemic inflammation and insulin resistance, is a risk factor for cancers and some other chronic diseases such as diabetes,

\footnotetext{
*Correspondence: aweizone@163.com; wangshouyu666@126.com ${ }^{+}$Wendong Wang and Mintao Zhong contributed equally to this work. 'Department of Orthopedic Surgery, The First Affiliated Hospital of Dalian Medical University, 222 Zhongshan Road, Dalian, China Full list of author information is available at the end of the article
}

atherosclerosis, fatty liver diseases [3-5]. High prevalence of obesity causes great damage to public health, however, many obesity-related therapeutic approaches, such as lifestyle changes, bariatric surgery, and pharmacotherapy, lead to an increased risk of various chronic diseases [6]. Finding novel therapeutic strategies is urgently necessary and challenging for prevention of obesity.

Increasing data indicates that gut microbiota composition is associated with obesity and its related metabolic disorders [7]. The gut microbiota composition varies significantly among obese and lean people [8]. For instance, an increase in the Firmicutes/Bacteroidetes (F/B) ratio 
can promote the development of obesity [9, 10]. Germfree mice with gut microbiota transplanted from high fat diet (HFD)-induced obese donors exhibited significantly increased body weight gain and metabolic syndromes [11]. In HFD-induced obese models, gut microbiota is also closely linked with intestinal permeability, which is associated with gut integrity and barrier function [12]. Meanwhile, gut microbiota plays an important role in nutrient acquisition, energy harvest and lipid metabolism, which are related to metabolic diseases [7, 13, 14]. Endotoxins, bile acids and short-chain fatty acids, produced by gut microbiota, link gut microbiota to metabolic health [15-18]. Therefore, modulating the composition of gut microbiota may be a potential new strategy for preventing obesity and its related metabolic disorders.

Recently, accumulating evidence has demonstrated that some bioactive ingredients, especially polysaccharides, can reduce obesity by modulating the composition of the gut microbiota $[9,19]$. For example, supplementation with polysaccharides isolated from sea cucumber or Ganoderma lucidum, reduced body weight gain and attenuated obesity-related metabolic syndromes in association with modulation of gut microbiota in HFD-fed mice $[9,19]$. These researches indicate that polysaccharides have beneficial effects on metabolism and gut microbiota.

WuGuChong, a traditional Chinese medicine with function of improving digestion disorders, has been used to treat infantile malnutrition and fulminant dysentery, as reported in traditional Chinese medical journals such as Compendium of Materia Medica and Materia Medica Companion [20]. Recently, extracts from WuGuChong have been demonstrated to have antibacterial effects, promote wound healing, and inhibit the progression of diabetes and atherosclerosis [21-23]. However, little is known about the effects of $W u G u C h o n g$ on gut microbiota, obesity and its related complications. In the present study, the effects of polysaccharide extracted from $\mathrm{WuGu}$ Chong (PEW) on HFD-induced obesity and obesityrelated liver steatosis, insulin resistance and systemic inflammation, as well as gut integrity were evaluated. Through the gut microbiota analysis, the results indicated that PEW had a beneficial regulation on the composition of gut microbiota, suggesting a potential mechanism by which PEW attenuated obesity and its related metabolic syndromes. Our study demonstrates that PEW may be a potential bioactive ingredient for preventing obesity.

\section{Methods}

\section{PEW preparation}

The dried bodies of $W u G u C h o n g$ were purchased in a traditional Chinese medicine market and identified according to the standards in "Chinese Materia Medica Standards". The PEW was isolated according to water extraction and alcohol precipitation methods as previous reported [24]. The Sevage method and DEAE iron-exchanged chromatography method were used for deproteinization and purification of polysaccharides. The PEW was characterized by GPC (gel permeation chromatogram) and HPLC (high performance liquid chromatography). The molecular weight of PEW was determined at $32.9 \mathrm{kDa}$, and its monosaccharide composition included rhamnose, glucose, mannose, galactose, arabinose, xylose, glucuronic acid, and galacturonic acid.

\section{Animal experiments}

Eight-week old C57BL/6 male mice were obtained from the Experimental Animal Center in Dalian Medical University in China. After 1 week of acclimating to the new surroundings, the mice were randomly divided into three groups $(n=9$ per group): The NCD (normal chow diet) group was conventionally fed a normal chow diet $(3.656 \mathrm{kcal} / \mathrm{g})$ containing $13.8 \% \mathrm{kcal}$ fat (Jiangsu Xietong Pharmaceutical Bioengineering Co., Ltd. China). The HFD group was fed a high fat diet $(4.73 \mathrm{kcal} / \mathrm{g}$ ) containing $45 \% \mathrm{kcal}$ fat (D12451, Jiangsu Xietong Pharmaceutical Bio-engineering Co., Ltd. China). The PEW treatment group (HFD + PEW) was fed a high fat diet (D12451) and given PEW $(300 \mathrm{mg} / \mathrm{kg})$ by oral gavage once a day. The other two groups also had oral gavage with water once a day. The experimental treatment continued for 8 weeks. The mice were maintained in a light and climate controlled room at $25 \pm 2{ }^{\circ} \mathrm{C}$ and $12 \mathrm{~h}$ light-dark cycle, with free access to water and different diets. Body weights and food intake were measured once a week. Liver and epididymal white adipose tissues (epi-WAT) were obtained and weighed immediately after treatment. The animal experimental protocols were approved by the ethics committee of Dalian Medical University (YJ-KY-SB-2019-83).

\section{Histopathological analysis}

The epi-WAT tissues were routinely fixed in $4 \%$ paraformaldehyde, processed for hematoxylin and eosin (HE) staining, and examined using a microscope (Tokyo Olympus, Japan). The liver tissues were analyzed with Oil Red O staining according to the protocol described by Chang et al. [9].

\section{Biochemical analysis}

Mice were fasted overnight after treatment, and blood samples were collected through the orbital sinus. The levels of total cholesterol (CHO), high and low density lipoprotein cholesterol (HDL-C and LDL-C), and triacylglycerol (TG) in the blood serum were measured using kits purchased from Nanjing Jiancheng Bioengineering Institute. Cytokine levels, including tumor necrosis factor- $\alpha$ (TNF- $\alpha$ ), interleukin-6 (IL-6), and lipopolysaccharide (LPS), were analyzed using ELISA kits (Shanghai Lengton Biological Technology Co., Ltd. China). 


\section{Glucose and insulin tolerance analysis}

The intraperitoneal glucose and insulin tolerance tests (IPGTT and ITT) were conducted at weeks 7 and 8, respectively. In brief, mice were either fasted overnight or for $6 \mathrm{~h}$, and then received an intraperitoneal injection of single-dose D-glucose $(1.0 \mathrm{~g} / \mathrm{kg})$ or insulin $(0.75 \mathrm{UI} / \mathrm{kg}$; Novo Nordisk). Blood from the caudal vein was collected at $0,15,30,60$, and $120 \mathrm{~min}$, respectively. A glucometer (Yuwell, Jiangsu, China) was used to measure blood glucose levels. Concentrations of fasting blood glucose and fasting serum insulin were measured, and the homeostasis model index of insulin resistance (HOMA-IR) was calculated according to the formula: HOMA-IR = insulin $\times$ glucose $/ 22.5$, as previously [25].

\section{Western blot analysis}

Protein samples were extracted from the ileum epithelial tissue using RIPA lysis buffer, and then quantified using a BCA protein quantification kit (KeyGEN BioTECH, China). Proteins were loaded onto an 8\% SDS-PAGE gel for electrophoresis, and then transferred onto PVDF membranes (Millipore, USA). After blocking with 5\% skimmed milk, membranes were incubated with primary antibodies against ZO-1 (Zonula occludin-1, 1:1000; Proteintech), occludin (1: 2000; Proteintech), and GAPDH (1:2000; Proteintech) over night at $4{ }^{\circ} \mathrm{C}$, and then with secondary antibody for $2 \mathrm{~h}$. Images were taken using $\mathrm{ChemiDoc}^{\text {rix }}$ MP imaging system (Bio-Rad, America).

\section{Gene transcription analysis}

Total RNA from ileum epithelial tissues was extracted using the TRIzol reagent (Invitrogen, USA) and reverse transcribed using the RevertAid First Strand cDNA Synthesis Kit (Thermo, USA). Real-time qPCR was conducted using the ABI 7500 Real-time PCR System (Applied Biosystems, USA). Values were normalized against $\beta$-actin and analyzed with the $2^{-\Delta \Delta C T}$ method. The real-time qPCR primers are listed in Table S1.

\section{Intestinal microbiota analysis}

Fresh stool samples obtained from the colon immediately upon sacrificing the mice were frozen in liquid nitrogen and stored at $-80^{\circ} \mathrm{C}$. A stool DNA Kit (D4015, Omega, USA) was used to isolate the fecal genomic DNA. The V3-V4 region of 16S rRNA (338F-806R, F: ACTCCTACGGGAGG CAGCAG; R: GGACTACHVGGGTWTCTAAT) was amplified for identification. The DNA template was initially denatured at $98^{\circ} \mathrm{C}$ for $30 \mathrm{~s}$, then amplified for 35 cycles of denaturation $\left(98^{\circ} \mathrm{C}\right.$ for $\left.10 \mathrm{~s}\right)$, annealing $\left(52^{\circ} \mathrm{C}\right.$ for $\left.30 \mathrm{~s}\right)$, and extension $\left(72^{\circ} \mathrm{C}\right.$ for $\left.45 \mathrm{~s}\right)$, and finally extended at $72{ }^{\circ} \mathrm{C}$ for $10 \mathrm{~min}$. The PCR products were purified by AMPure XT beads (Beckman Coulter Genomics, Danvers, MA, USA) and quantified by Qubit (Invitrogen, USA). The amplicon pools were prepared for sequencing and the size and quantity of the amplicon library were assessed on Agilent 2100 Bioanalyzer (Agilent, USA) and with the Library Quantification Kit for Illumina (Kapa Biosciences, Woburn, MA, USA), respectively. The PCR products were sequenced on an Illumina MiSeq platform (LC-Bio Technology Co., Ltd., Hang Zhou, China). Paired-end reads was assigned to samples based on their unique barcode and truncated by cutting off the barcode and primer sequence. Paired-end reads were merged using FLASH [26]. Quality filtering on the raw tags were performed under specific filtering conditions to obtain the high-quality clean tags according to the fqtrim (V 0.94). Chimeric sequences were filtered using Vsearch software (v2.3.4). Sequences with $\geq 97 \%$ similarity were assigned to the same operational taxonomic units (OTUs) by Vsearch (v2.3.4) [27]. Representative sequences were chosen for each OTU, and taxonomic data were then assigned to each representative sequence using the RDP (Ribosomal Database Project) classifier. The differences of the dominant species in different groups, multiple sequence alignment were conducted using the mafft software (V 7.310) to study phylogenetic relationship of different OTUs. OTUs abundance information were normalized using a standard of sequence number corresponding to the sample with the least sequences. Alpha diversity is applied in analyzing complexity of species diversity for a sample through 5 indices, including Chao1, Observed species, Goods coverage, Shannon, and Simpson. All the indices in the samples were calculated with QIIME (Version 1.8.0). Beta diversity analysis was used to evaluate differences of samples in species complexity. For beta-diversity analysis, principal coordinate analysis ( $\mathrm{PCoA})$ and unweighted pair group method with arithmetic mean (UPGMA) clustering were performed by QIIME software (Version 1.8.0) [28]. The linear discriminant analysis (LDA) effect size (LEfSe) analysis was conducted for quantitative analysis of biomarkers in different groups [29]. Briefly, Kruskal-Wallis rank-sum test and Wilcoxon rank rank-sum test were used for LEfSe analysis $(P<0.05$; LDA $>3.0)$, to identify the most differently abundant taxa.

\section{Statistical analysis}

All data are presented as means \pm SD. The SPSS statistics 23.0 software (IBM, USA) was used for statistical analysis. Differences across groups were analyzed using one-way ANOVA followed by Tukey's multiple comparison tests. Differences were considered as statistically significant if $P<0.05$.

\section{Results}

PEW reduces body weight in HFD-treated mice

To determine the influence of PEW on body weight in HFD-treated mice, eight-week old C57BL/6 male mice were divided into three groups: NCD group, HFD group, HFD + PEW group. As expected, the HFD group exhibited increased body weight gain compared to the NCD group, which was reduced by PEW supplementation (Fig. 1a-b). 

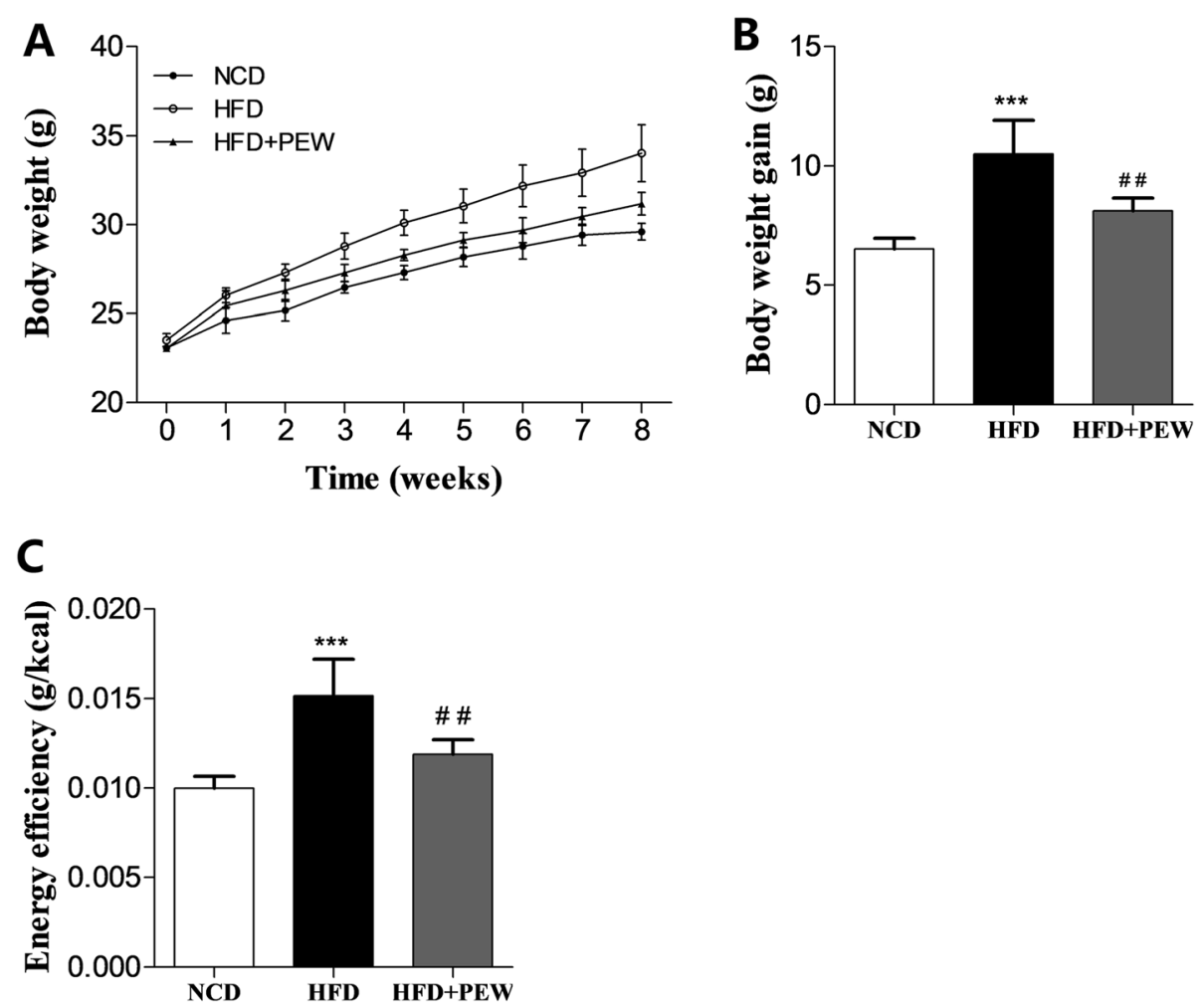

Fig. 1 Effects of PEW on body weight ( $n=5$ for each group). a Body weight versus time analysis; b Body weight gain; c Energy efficiency, calculated according to the formula: body weight gain/energy intake. Data are presented as means $\pm S D$, and analyzed using the one-way ANOVA test with Tukey method. ${ }^{* * *} P<0.001$ compared with NCD; ${ }^{\# \#} P<0.05$ compared with HFD

Compared with the NCD group, the energy efficiency (weight gain divided by energy intake) in the HFD group was remarkably increased. Supplementation with PEW significantly decreased the energy efficiency in the HFD-fed mice (Fig. 1c), suggesting lower weight gain per kcal energy intake.

\section{PEW inhibits liver steatosis and adipose hypertrophy in HFD-treated mice}

Epi-WAT and liver weights were first measured to evaluate the effect of PEW on tissue changes. The epi-WAT weight was significantly induced by the HFD and reversed by PEW supplementation, while liver weight was not affected by HFD and PEW supplementation (Fig. 2a-b). Moreover, the HFD group exhibited larger adipocyte cells compared to the NCD group and PEW supplementation group (Fig. 2c), demonstrating that PEW prevents HFD-induced adipose hypertrophy. Compared to the NCD and PEW supplementation groups, fat accumulation in the liver was increased in the HFD group as indicated by Oil Red O staining (Fig. 2d), suggesting that PEW might attenuate liver steatosis.

PEW attenuates serum lipids, cholesterol levels, insulin resistance, and glucose tolerance

To assess the ability of PEW to attenuate hypercholesterolemia and hyperlipidemia, we measured serum lipid concentrations in mice. The HFD group had a significant increase in TG, CHO, HDL-C, and LDL-C concentrations (48.2, 89.2, 86.9, and 270.8\%, respectively) compared to the NCD group (Fig. 3a-d). Supplementation with PEW significantly decreased the $\mathrm{CHO}, \mathrm{HDL}-\mathrm{C}$, and LDL-C concentrations $(-22.7,-23.0$, and $-35.2 \%$, respectively) in the HFD-fed mice. However, the TG concentration was not significantly affected by PEW supplementation. These results suggested that PEW might attenuate hypercholesterolemia and hyperlipidemia in HFD-fed mice.

As obesity is closely correlated with insulin resistance and glucose tolerance [30], fasting blood glucose and fasting serum insulin was measured, and HOMA-IR was calculated according to the established formula. In addition, IPGTT and ITT tests were also performed in the present study. Supplementation with PEW maintained insulin sensitivity in HFD-fed mice, and improved fasting blood glucose, fasting serum insulin and HOMAIR, which were closed to the control levels (Fig. 3e-g). As shown in Fig. 3h-i, compared with the NCD or PEW supplementation groups, the HFD group exhibited higher IPGTT, ITT, and AUC (area under curve) values, further suggesting that PEW supplementation significantly decreased the induced glucose tolerance and insulin resistance in HFD-treated mice. 

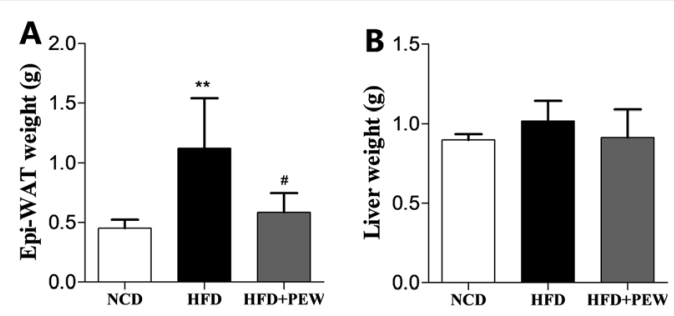

C

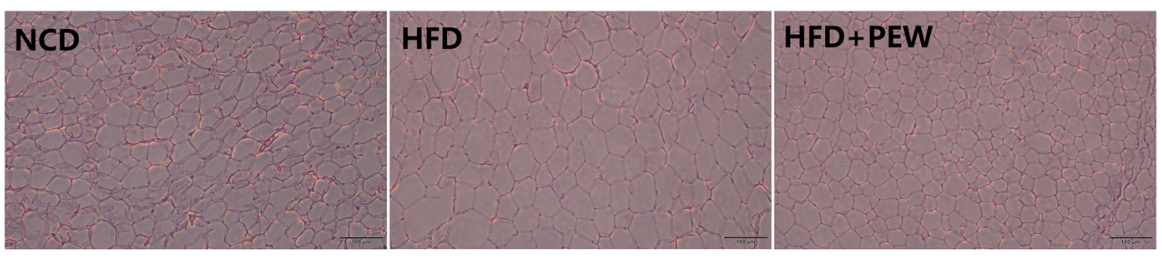

D

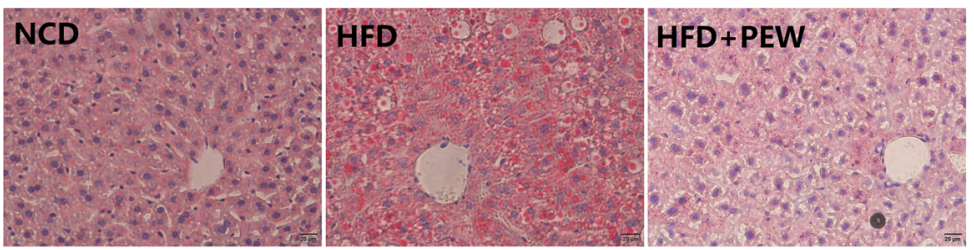

Fig. 2 Effects of PEW on adipose hypertrophy and liver steatosis ( $n=5$ for each group). a epi-WAT weight; b Liver tissue weight. c HE staining of epi-WAT tissues (scale bar $=100 \mu \mathrm{m}$ ); $\mathbf{d}$ Oil red O staining of liver tissues (scale bar $=20 \mu \mathrm{m}$ ). Data are presented as means \pm SD, and analyzed using the one-way ANOVA test with Tukey method; ${ }^{* *} P<0.01$ compared with NCD; ${ }^{\#} P<0.05$ compared with HFD

\section{PEW prevents HFD-induced systemic inflammation}

It was previously reported that obesity and liver steatosis are accompanied by increased levels of serum endotoxins (LPS) and inflammatory cytokines $[9,31]$. The effects of PEW on LPS release and cytokines secretion were examined in this study. As expected, compared with the NCD group, the HFD group displayed a significant increase in serum LPS, TNF- $\alpha$, and IL- 6 levels, which was reversed by PEW supplementation (Fig. 4a-c), suggesting that PEW might prevent HFD-induced endotoxemia and systemic inflammation.

\section{PEW maintains intestinal epithelium integrity}

The effects of PEW on the integrity of the gut epithelium were evaluated by analyzing the expression of gut integrity biomarkers occludin and ZO-1 [32]. HFD strongly suppressed occludin and ZO-1 mRNA levels as identified by RT-PCR analysis (Fig. 5a-b). PEW supplementation significantly increased occludin levels but not ZO-1 in HFD-fed mice. However, the reduced occludin and ZO-1 protein expression in HFD-fed mice was recovered by PEW supplementation as shown by Western blot analysis (Fig. 5c). The results suggested that PEW supplementation maintained the gut integrity and barrier function.

\section{PEW alters the intestinal microbiota composition in HFD- fed mice}

The intestinal microbiota composition was determined by sequencing the $16 \mathrm{~S}$ rRNA (V3 $+\mathrm{V} 4$ region) using the
Illumina MiSeq platform. Based on 97\% identity level, OTU averages of 2511, 3561, and 3478 were respectively clustered in the NCD, HFD, and HFD + PEW groups. As shown in Fig. S1A, the OTUs in the HFD group were higher compared to the NCD group, while PEW supplementation did not significantly decrease the OTUs. The curves of OTU rank, Chao 1, Shannon, and Simpson are presented in Fig. S1B, C, D, and E, respectively. The indexes of Chao 1 (Fig. S1F), Shannon (Fig. S1G), and Simpson (Fig. S1H) were calculated. The curves of OTU rank and Chao 1 were consistent with the number of OTUs. No significant difference was found for Shannon and Simpson indexes, suggesting that there was no difference in richness and diversity of intestinal microbiota between the three groups.

As shown in Fig. 6a, the relative decreased abundance of Bacteroidetes and increased abundance of Firmicutes and Proteobacteria were observed in the HFD group in the phylum level, and the F/B ratio was also increased in the HFD group compared to the NCD group. PEW supplementation restored the levels of Bacteroidetes, Firmicutes, and Proteobacteria, and significantly decreased the F/B ratio (Fig. 6b). The difference of genus level among each group was further investigated, and the results were consistent with the phylum level. PEW supplementation significantly increased the relative abundance of Parabacteroides belonging to the Bacteroidetes phylum, and significantly decreased the relative abundance of 

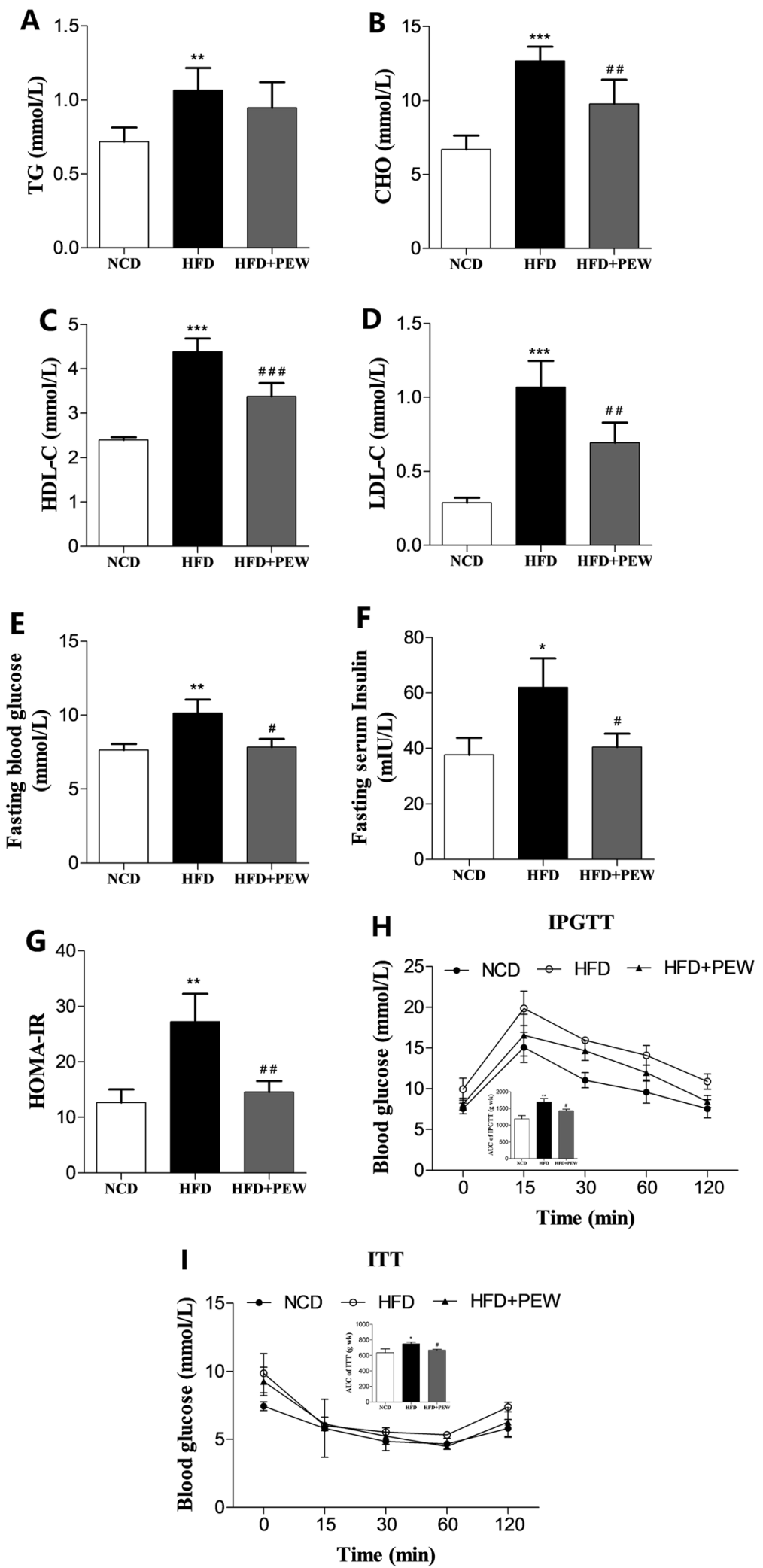

Fig. 3 (See legend on next page.) 
(See figure on previous page.)

Fig. 3 Effects of PEW on lipid concentration, glucose tolerance, and insulin resistance ( $n=5$ for each group). a-d TG (triacylglycerol), $C H O$ (cholesterol), HDL-C (high density lipoprotein cholesterol), and LDL-C (low density lipoprotein cholesterol) levels in NCD, HFD, and HFD + PEW groups; e-g Fasting blood glucose, fasting serum insulin and HOMA-IR, calculated according to the formula: HOMA-IR=insulin $\times$ glucose/22.5. $\mathbf{h}-\mathbf{i}$ Blood glucose versus time after intraperitoneal injection of D-glucose or insulin. The calculated AUC (inset) are also shown. Data are presented as means $\pm \mathrm{SD}$, and analyzed using the one-way ANOVA test with Tukey method. ${ }^{*} P<0.05,{ }^{* *} P<0.01,{ }^{* * *} P<0.001$ compared with $N C D ;{ }^{\#} P<0.05$, $\# P<0.01, \# \# P<0.001$ compared with HFD

Ihubacter, Lachnospiraceae_unclassified, and Lachnostridium belonging to the Firmicutes phylum, as well as Bilophila belonging to the Proteobacteria phylum in HFD-treated mice (Fig. 6c). In addition, unweighted unifrac cluster based UPGMA and the unweighted and weighted unifrac distance based PCoA were performed to investigate gut microbiota structural changes. As shown in Fig. 6d-f, all three groups exhibited distinctive microbiota profiles. Furthermore, the HFD + PEW group and HFD group showed a similar microbiota structure.

The biomarkers in the gut microbiota sequences were analyzed using the LEfSe method. Compared with the HFD group, the successive circles and bar graph showed that 50 phylotypes were higher and 90 were lower in the NCD group, and 21 phylotypes were higher and 21 were lower in the HFD + PEW group (Fig. 7a-d). The results indicate that PEW supplementation significantly increased the abundance of beneficial phylotypes Bacteroidetes, and decreased the abundance of pathogenic phylotypes Firmicutes and Proteobacteria in HFD-treated mice. As shown in Fig. 7e, PEW supplementation induced an increase in Clostridium_IV, which had a potential probiotic effect [33]. The abundance of pathogenic bacteria, including Desulfovibrionaceae, Lachnospiraceae, and Bilophila [3436], were reduced after PEW supplementation in HFDtreated mice (Fig. 7f-h). These results indicated that PEW supplementation might prevent HFD-induced intestinal microbiota dysbiosis.

\section{Discussion}

Gut microbiota is closely associated with obesity and its related metabolic disorders [7]. Recent research data show that polysaccharides extracted from natural products prevent HFD-induced obesity and its related complications by modulating the composition of gut microbiota $[9,19]$. However, the effects of PEW on obesity and gut microbiota has not been investigated. In the study, we investigated the effects of PEW supplementation for 8 weeks on HFD-induced obesity and gut microbiota. The results demonstrate that PEW could prevent HFD-induced
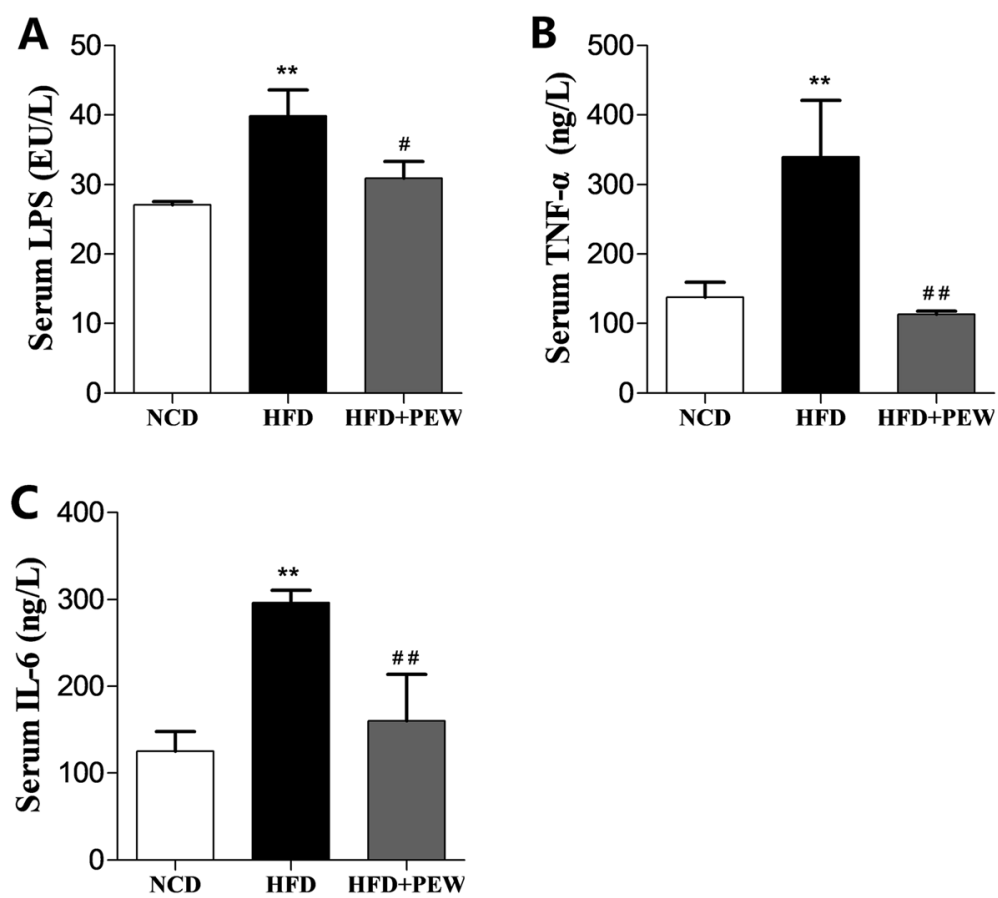

Fig. 4 Effects of PEW on systemic inflammation ( $n=5$ for each group). a Serum level of endotoxins; $\mathbf{b}$ Serum level of TNF-a; c Serum level of IL-6. Data are presented as means $\pm \mathrm{SD}$, and analyzed using the one-way ANOVA test with Tukey method. ${ }^{* *} P<0.01$ compared with $N C D$; ${ }^{\#} P<0.05$, ${ }^{\# \#} P<0.01$ compared with HFD 

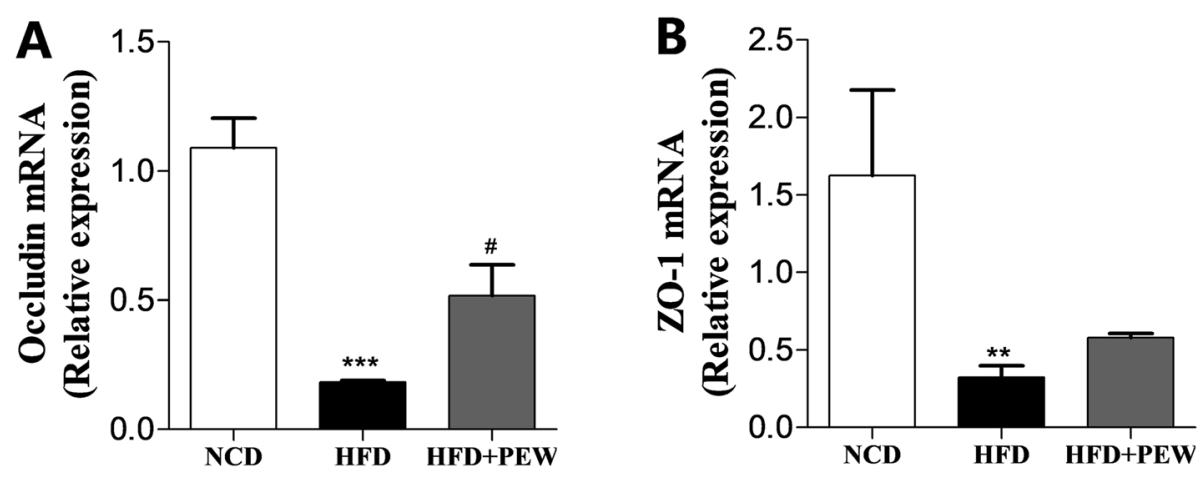

C
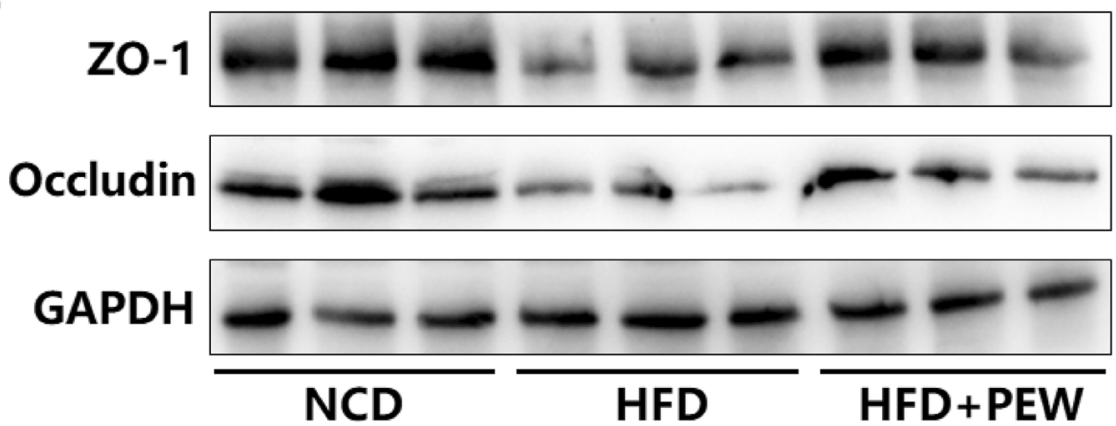

Fig. 5 Relative expression of occludin and ZO-1 ( $n=3$ for each group). a-b RT-qPCR analysis of the relative mRNA levels of occludin and ZO-1; c Western blot analysis of occludin and ZO-1. Data are presented as means \pm SD, and analyzed using the one-way ANOVA test with Tukey method. ${ }^{* *} P<0.01,{ }^{* * *} P<0.001$ compared with NCD; ${ }^{\#} P<0.05$ compared with HFD

obesity, and its related liver steatosis, insulin resistance and systemic inflammation by modulating the composition of gut microbiota. In addition, PEW supplementation could also maintain the intestinal epithelium integrity.

In the present study, PEW supplementation significantly prevents body weight gain, adipose hypertrophy in HFD-fed mice (Fig. 1a-b and Fig. 2a, c). As previously reported, the composition of gut microbiota in obese and lean individuals was significantly different [8]. In the gut microbiota of obese models, increased abundances of Firmicutes and decreased abundances of Bacteroidetes were observed $[9,37]$. Our results show that HFD increases the abundance of Firmicutes and decreases the abundance of Bacteroidetes. Consistent with the Chang's research [9], PEW supplementation significantly reverses the changes, and decreases the increased F/B ratio in HFD-fed mice to approximately the control levels (Fig. 6a-b), which may contribute to its anti-obesity effects. Additionally, at the phylum level, endotoxin-bearing Proteobacteria is also decreased by PEW supplementation. Obesity is often accompanied by fat accumulation in the viscera, especially in liver, and closely associated with chronic systemic inflammation, which may induce many chronic diseases [38]. As expected, PEW supplementation attenuates liver steatosis induced by HFD in the study (Fig. 2d). Previous evidence indicated that significantly increased inflammatory cytokine
TNF- $\alpha$ and IL- 6 levels were observed in obese individuals [39]. The pro-inflammatory molecules can affect the host metabolic process, as TNF- $\alpha$ was reported to decrease the insulin sensitivity and increase lipolysis in adipocytes, as well as IL-6 was found to contribute to hypertriglyceridemia [40, 41]. In our study, serum levels of TNF- $\alpha$ and IL-6, lipids, cholesterol, glucose and insulin increased significantly in HFD group (Fig. 3 and Fig. 4b-c). However, PEW supplementation significantly reduces systemic inflammation, insulin resistance, hypercholesterolemia and hyperlipidemia in HFD-fed mice. The results suggest that PEW improves obesity-related metabolic disorders by modulating systemic inflammation.

Accumulating evidence suggests that gut microbiota is closely linked to host systemic inflammation, which is a feature of obesity [39, 42]. Significantly increased inflammatory cytokine TNF- $\alpha$ and IL- 6 , as well as LPS, all markers of systemic inflammation, were observed in obese models as previous report [43], and decreased by PEW supplementation (Fig. 4a-c). Gut microbiota dysbiosis, which contributes to LPS production, is the potential mechanism for systemic inflammation induced by HFD. Endotoxins, known as LPS, which induced the release of proinflammatory cytokine, were predominantly derived from Gram-negative bacteria in intestinal microbiota $[44,45]$. In the present study, the relative abundance of endotoxin- 


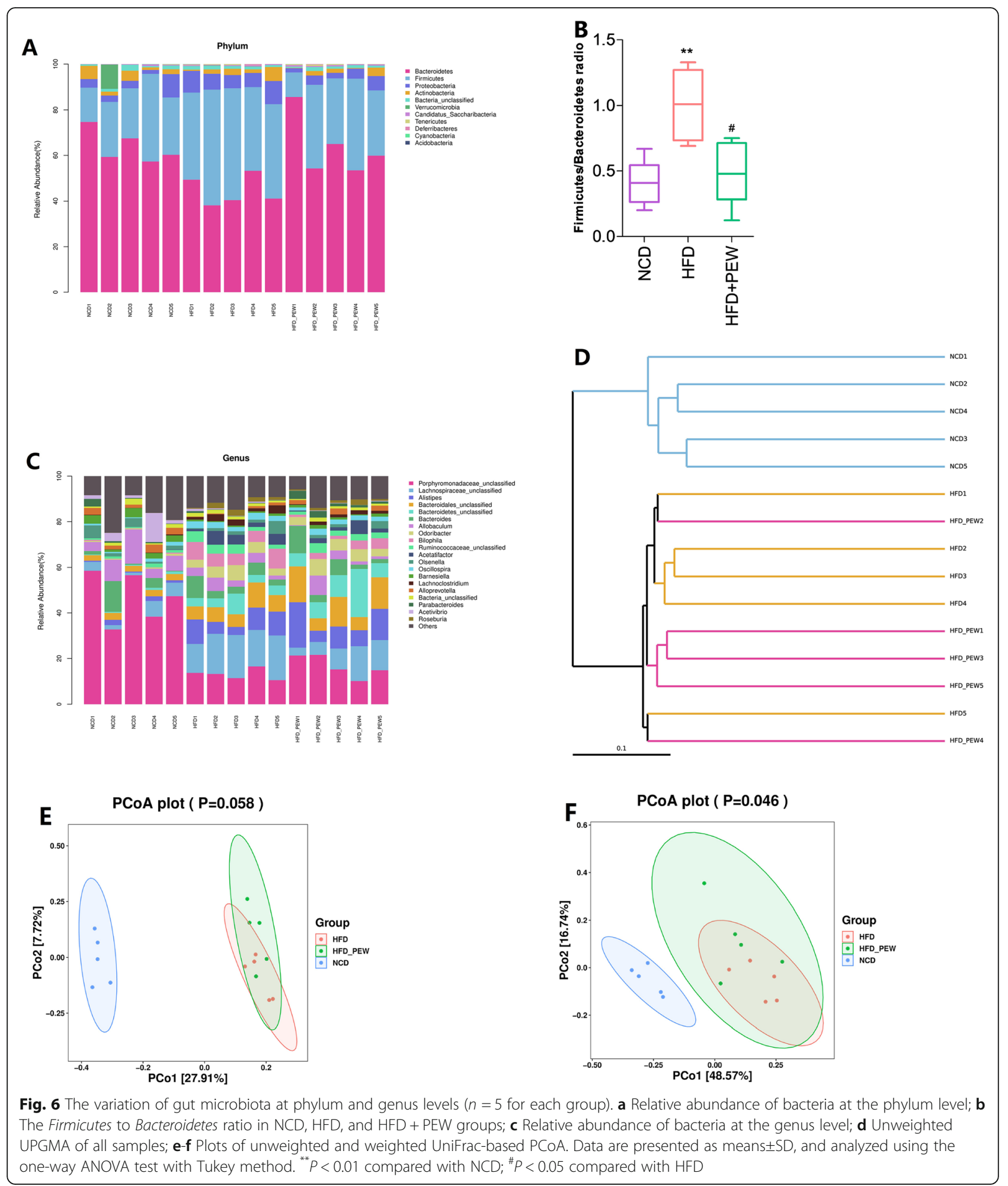

producing bacteria Desulfovibrionaceae is increased in HFD group, and reversed by PEW supplementation (Fig. 7f). The results are in agreement with previous research, which demonstrated that the relative abundance of Desulfovibrionaceae was decreased by dietary intervention in obese individuals
[34]. The relative abundance of Bilophila has been demonstrated to be positively related to obesity and inflammation [36]. In the present study, the relative abundance of Bilophila is increased by HFD, and restored by PEW supplementation (Fig. 7h), which is consistent with the previous report. Recent 


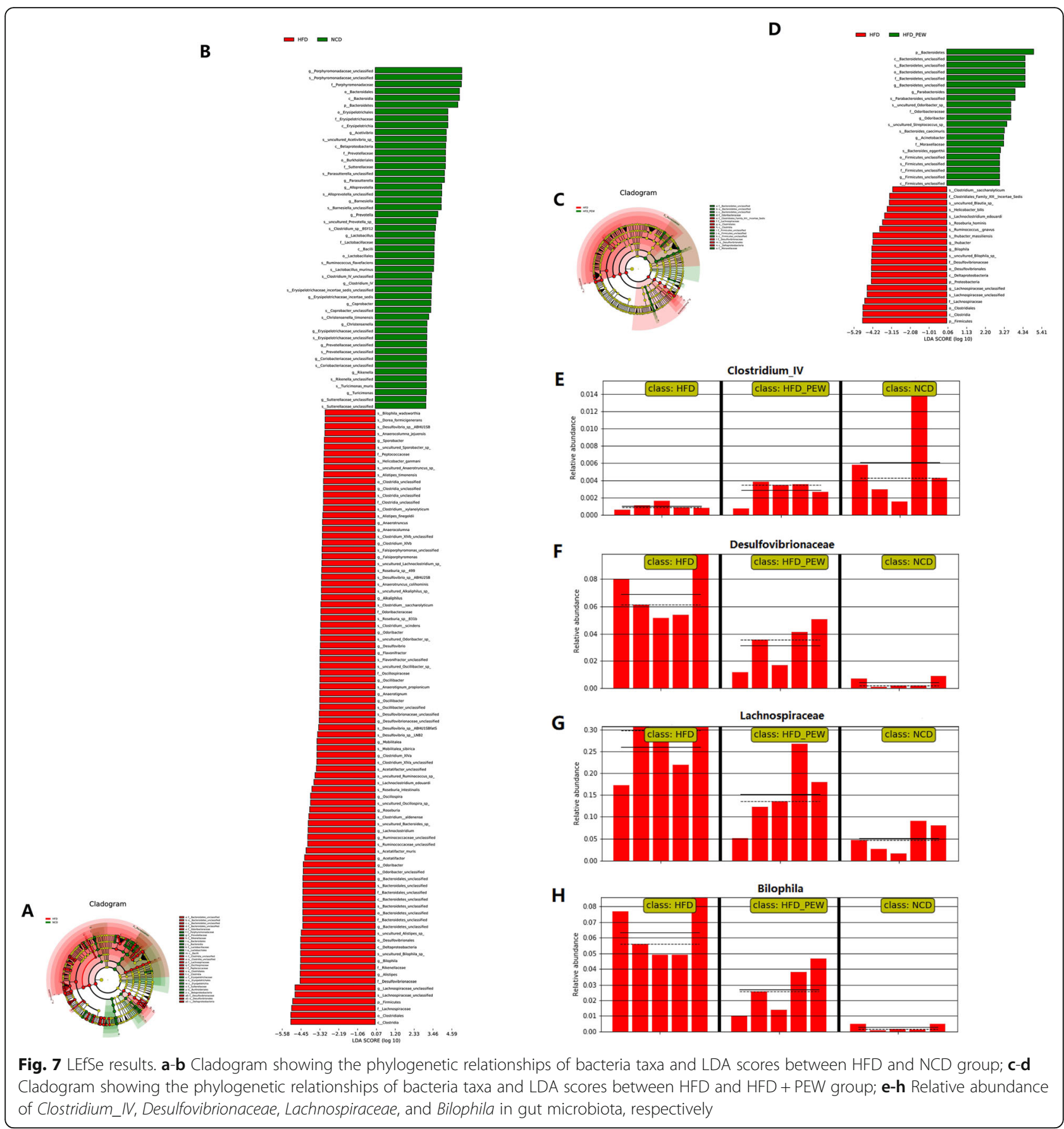

research observed that the relative abundance of Lachnospiraceae was increased in high-fat/high-sucrose (HFHS)-fed mice [35]. Here, PEW supplementation significantly decreases the relative abundance of Lachnospiraceae, which is increased by HFD (Fig. 7g). In addition, Clostridium_IV, belonging to Clostridium clusters, which lacks prominent toxins and virulence factors, has potential probiotic effects. Meanwhile, Clostridium_IV was also reported to regulate host fatty acid metabolism, immune function and ameliorated colitis [33]. Our study demonstrates that PEW supplementation enhances the relative abundance of Clostridium_IV in HFDtreated mice (Fig. 7e). The results suggest that the antiobesity and anti-inflammation effects of PEW may be due to modulation of the gut microbiota composition.

Previous study reported that HFD-induced gut microbiota dysbiosis contributed to the damage of intestinal epithelium integrity, and the release of LPS into circulation, resulting in insulin resistance, systemic inflammation and obesity [9]. Otherwise, gut microbiota could also help strengthen mucosal defense by promoting epithelial 
renewal and immune maturation, as well as decreasing intestinal permeability $[46,47]$. In the study, PEW supplementation increases the expression of occludin and ZO-1, which are decreased by HFD (Fig. 5), resulting in maintaining intestinal epithelium integrity. This is consistent with the previous study, which reported that increased expression of occludin and ZO-1, along with modulation of the gut microbiota composition, contributed to strengthen the gut integrity and barrier function [48]. The results suggest that PEW may improve obesity-related metabolic disorders by modulating gut microbiota and maintaining intestinal epithelium integrity.

In conclusion, our results show that PEW supplementation prevents HFD-induced obesity and its related metabolic disorders, including liver steatosis, insulin resistance and systemic inflammation, by modulating gut microbiota composition and maintaining intestinal epithelium integrity. PEW supplementation decreases Firmicutes to Bacteroidetes ratio and the relative abundance of Proteobacteria in HFD-fed mice, contributing to the beneficial effects against obesity and its related disorders. Our study suggests that PEW may be used as a bioactive ingredient to prevent obesity. However, the detailed mechanisms need to be further investigated.

\section{Supplementary information}

Supplementary information accompanies this paper at https://doi.org/10. 1186/s12986-020-00442-2.

Additional file 1: Table S1. Primers used in this study.

Additional file 2: Figure S1. The characteristics of gut microbiota. (A) OTU clusters of gut microbiota; (B-E) OTU rank curves, Chao 1 curves, Shannon curves, and Simpson curves of gut microbiota, respectively; ( $F$ H) Chao 1, Shannon, and Simpson indexes of gut microbiota,

respectively. Data are presented as means and standard deviation, and analyzed using the one-way ANOVA test. ${ }^{* *} P<0.01$ compared with NCD.

\section{Abbreviations}

CHO: Total cholesterol; epi-WAT: Epididymal white adipose tissues; GPC: Gel permeation chromatogram; HE: Hematoxylin and eosin; HFD: High-fat diet; IPGTT: Intraperitoneal glucose test; ITT: Insulin tolerance test; LDA: Linear discriminant analysis; NCD: Normal chow diet; OTU: Operational taxonomic unit; PEW: Polysaccharide extracted from WuGuChong; ZO-1: Zonula occludin-1

\section{Acknowledgements}

Not applicable.

\section{Authors' contributions}

Junwei Zong and Shouyu Wang contributed to the study's conception and design. Wendong Wang and Mintao Zhong contributed to drafting of the manuscript. Wendong Wang, Mintao Zhong, Tiantina Yu, Lei Chen and Lijun Shi performed experiments and data collection. Wendong Wang and Mintao Zhong performed data analysis and interpretation. The final draft was read and approved by all the authors.

\section{Funding}

This work was supported by National Natural Science Foundation of China (81573734), Liaoning Revitalization Talents Program (XLYC1802014), Liaoning Key Research and Development Planning Project (2017226015),

Distinguished Professor Project of Liaoning Province, Liaoning BaiQianWan
Talents Program and Clinical Capability Construction Project for Liaoning Provincial Hospitals (LNCCC-A04-2014).

\section{Availability of data and materials}

The datasets used and/or analyzed during the current study are available from the corresponding author on reasonable request.

\section{Ethics approval}

This animal study was approved by the ethics committee of Dalian Medical University (YJ-KY-SB-2019-83).

\section{Consent for publication}

Not applicable.

\section{Competing interests}

The authors declare that they have no competing interests.

\section{Author details}

'Department of Orthopedic Surgery, The First Affiliated Hospital of Dalian Medical University, 222 Zhongshan Road, Dalian, China. ${ }^{2}$ College of Integrative Medicine, Dalian Medical University, 9 South Lushun Road West, Dalian, China. ${ }^{3}$ Department of Microbiology, Dalian Medical University, 9 South Lushun Road West, Dalian, China. ${ }^{4}$ Department of Gynaecology and Obstetrics, Dalian Municipal Woman and Children's Medical Center, 1 and 3 Sports new town first Road, Dalian, China.

Received: 20 December 2019 Accepted: 12 March 2020

Published online: 30 March 2020

\section{References}

1. Cecchini M, Sassi F, Lauer JA, Lee YY, Guajardo-Barron V, Chisholm D. Tackling of unhealthy diets, physical inactivity, and obesity: health effects and cost-effectiveness. Lancet. 2010;376:1775-84

2. Morgen CS, Sorensen TI. Obesity: global trends in the prevalence of overweight and obesity. Nat Rev Endocrinol. 2014;10:513-4.

3. Arnold M, Pandeya N, Byrnes G, Renehan PAG, Stevens GA, Ezzati PM, Ferlay J, Miranda JJ, Romieu I, Dikshit R, et al. Global burden of cancer attributable to high body-mass index in 2012: a population-based study. Lancet Oncol. 2015;16:36-46.

4. Yoshimoto S, Loo TM, Atarashi K, Kanda H, Sato S, Oyadomari S, Iwakura Y, Oshima K, Morita H, Hattori M, et al. Obesity-induced gut microbial metabolite promotes liver cancer through senescence secretome. Nature. 2013;499:97-101.

5. Osborn O, Olefsky JM. The cellular and signaling networks linking the immune system and metabolism in disease. Nat Med. 2012;18:363-74.

6. Heymsfield SB, Wadden TA. Mechanisms, pathophysiology, and management of obesity. N Engl J Med. 2017;376:1492.

7. Turnbaugh PJ, Ley RE, Mahowald MA, Magrini V, Mardis ER, Gordon Jl. An obesity-associated gut microbiome with increased capacity for energy harvest. Nature. 2006:444:1027-31.

8. Allen JM, Mailing L, Niemiro GM, Moore R, Cook MD, White BA, Holscher HD, Woods JA. Exercise alters gut microbiota composition and function in lean and obese humans. Med Sci Sports Exerc. 2018;50:747-57.

9. Chang CJ, Lin CS, Lu CC, Martel J, Ko YF, Ojcius DM, Tseng SF, Wu TR, Chen YY, Young JD, Lai HC. Ganoderma lucidum reduces obesity in mice by modulating the composition of the gut microbiota. Nat Commun. 2015;6:7489.

10. Ley RE, Backhed F, Turnbaugh P, Lozupone CA, Knight RD, Gordon Jl. Obesity alters gut microbial ecology. Proc Natl Acad Sci U S A. 2005;102: $11070-5$.

11. Turnbaugh PJ, Backhed F, Fulton L, Gordon JI. Diet-induced obesity is linked to marked but reversible alterations in the mouse distal gut microbiome. Cell Host Microbe. 2008;3:213-23.

12. Rohr MW, Narasimhulu CA, Rudeski-Rohr TA, Parthasarathy S. Negative effects of a high-fat diet on intestinal permeability: a review. Adv Nutr. 2020;11:77-91.

13. Turnbaugh PJ, Hamady M, Yatsunenko T, Cantarel BL, Duncan A, Ley RE, Sogin ML, Jones WJ, Roe BA, Affourtit JP, et al. A core gut microbiome in obese and lean twins. Nature. 2009;457:480-4.

14. Tremaroli V, Backhed F. Functional interactions between the gut microbiota and host metabolism. Nature. 2012;489:242-9.

15. Janssen AW, Kersten S. Potential mediators linking gut bacteria to metabolic health: a critical view. J Physiol. 2017;595:477-87. 
16. Koh A, De Vadder F, Kovatcheva-Datchary P, Backhed F. From dietary fiber to host physiology: short-chain fatty acids as key bacterial metabolites. Cell. 2016;165:1332-45.

17. Sayin SI, Wahlstrom A, Felin J, Jantti S, Marschall HU, Bamberg K, Angelin B, Hyotylainen T, Oresic M, Backhed F. Gut microbiota regulates bile acid metabolism by reducing the levels of tauro-beta-muricholic acid, a naturally occurring fxr antagonist. Cell Metab. 2013;17:225-35.

18. Cani PD, Amar J, Iglesias MA, Poggi M, Knauf C, Bastelica D, Neyrinck AM, Fava F, Tuohy KM, Chabo C, et al. Metabolic endotoxemia initiates obesity and insulin resistance. Diabetes. 2007:56:1761-72.

19. Zhu Z, Zhu B, Sun Y, Ai C, Wang L, Wen C, Yang J, Song S, Liu X. Sulfated polysaccharide from sea cucumber and its depolymerized derivative prevent obesity in association with modification of gut microbiota in highfat diet-fed mice. Mol Nutr Food Res. 2018;62:e1800446.

20. Gao J, Li W, Niu L, Cao R, Yin W. Isolation and structural elucidation of novel antimicrobial compounds from maggots of chrysomyis megacephala fabricius. Nat Prod Res. 2015;29:239-46.

21. Zhang Z, Wang J, Zhang B, Liu H, Song W, He J, Lv D, Wang S, Xu X. Activity of antibacterial protein from maggots against staphylococcus aureus in vitro and in vivo. Int J Mol Med. 2013;31:1159-65.

22. Zhang Z, Wang S, Diao Y, Zhang J, Lv D. Fatty acid extracts from lucilia sericata larvae promote murine cutaneous wound healing by angiogenic activity. Lipids Health Dis. 2010;9:24

23. Mei H, Xu J, He Y, Yang X, Liu W, Tian W, Zeng YU, Zhu J. Protein-rich extract of musca domestica larvae alleviated metabolic disorder in stzinduced type 2 diabetic rat model via hepatoprotective and pancreatic beta-cell protective activities. J Biosci. 2018;43:969-83.

24. Gao H, Wen JJ, Hu JL, Nie QX, Chen HH, Xiong T, Nie SP, Xie MY. Polysaccharide from fermented momordica charantia I. with lactobacillus plantarum ncu116 ameliorates type 2 diabetes in rats. Carbohydr Polym. 2018;201:624-33.

25. Xu P, Hong F, Wang J, Cong Y, Dai S, Wang S, Wang J, Jin X, Wang F, Liu J, Zhai $Y$. Microbiome remodeling via the montmorillonite adsorption-excretion axis prevents obesity-related metabolic disorders. EBioMedicine. 2017;16:251-61.

26. Magoc T, Salzberg SL. Flash: fast length adjustment of short reads to improve genome assemblies. Bioinformatics. 2011;27:2957-63.

27. Edgar RC. Search and clustering orders of magnitude faster than blast. Bioinformatics. 2010;26:2460-1.

28. Caporaso JG, Kuczynski J, Stombaugh J, Bittinger K, Bushman FD, Costello EK, Fierer N, Pena AG, Goodrich JK, Gordon Jl, et al. Qiime allows analysis of high-throughput community sequencing data. Nat Methods. 2010;7:335-6.

29. Segata N, Izard J, Waldron L, Gevers D, Miropolsky L, Garrett WS, Huttenhower C. Metagenomic biomarker discovery and explanation. Genome Biol. 2011;12:R60.

30. Atamni HJ, Mott R, Soller M, Iraqi FA. High-fat-diet induced development of increased fasting glucose levels and impaired response to intraperitoneal glucose challenge in the collaborative cross mouse genetic reference population. BMC Genet. 2016;17:10

31. Harte AL, da Silva NF, Creely SJ, McGee KC, Billyard T, Youssef-Elabd EM, Tripathi G, Ashour E, Abdalla MS, Sharada HM, et al. Elevated endotoxin levels in non-alcoholic fatty liver disease. J Inflamm (Lond). 2010;7:15.

32. Odenwald MA, Turner JR. The intestinal epithelial barrier: a therapeutic target? Nat Rev Gastroenterol Hepatol. 2017;14:9-21.

33. Atarashi K, Tanoue T, Oshima K, Suda W, Nagano Y, Nishikawa H, Fukuda S, Saito T, Narushima S, Hase K, et al. Treg induction by a rationally selected mixture of clostridia strains from the human microbiota. Nature. 2013;500:232-6.

34. Xiao S, Fei N, Pang X, Shen J, Wang L, Zhang B, Zhang M, Zhang X, Zhang $C$, Li $M$, et al. A gut microbiota-targeted dietary intervention for amelioration of chronic inflammation underlying metabolic syndrome. FEMS Microbiol Ecol. 2014;87:357-67.

35. Masumoto S, Terao A, Yamamoto Y, Mukai T, Miura T, Shoji T. Nonabsorbable apple procyanidins prevent obesity associated with gut microbial and metabolomic changes. Sci Rep. 2016;6:31208.

36. Wang J, Tang H, Zhang C, Zhao Y, Derrien M, Rocher E, van- Hylckama Vlieg JE, Strissel K, Zhao L, Obin M, Shen J. Modulation of gut microbiota during probiotic-mediated attenuation of metabolic syndrome in high fat diet-fed mice. ISME J. 2015:9:1-15.

37. Ley RE, Turnbaugh PJ, Klein S, Gordon Jl. Microbial ecology: human gut microbes associated with obesity. Nature. 2006;444:1022-3.

38. Rinella ME. Nonalcoholic fatty liver disease: a systematic review. JAMA. 2015; 313:2263-73.
39. Kang Y, Li Y, Du Y, Guo L, Chen M, Huang X, Yang F, Hong J, Kong X. Konjaku flour reduces obesity in mice by modulating the composition of the gut microbiota. Int J Obes. 2019;43:1631-43.

40. Zhang HH, Halbleib M, Ahmad F, Manganiello VC, Greenberg AS. Tumor necrosis factor-alpha stimulates lipolysis in differentiated human adipocytes through activation of extracellular signal-related kinase and elevation of intracellular camp. Diabetes. 2002;51:2929-35.

41. Nonogaki K, Fuller GM, Fuentes NL, Moser AH, Staprans I, Grunfeld C, Feingold KR. Interleukin-6 stimulates hepatic triglyceride secretion in rats. Endocrinology. 1995;136:2143-9.

42. Cani PD, Bibiloni R, Knauf C, Waget A, Neyrinck AM, Delzenne NM, Burcelin R. Changes in gut microbiota control metabolic endotoxemia-induced inflammation in high-fat diet-induced obesity and diabetes in mice. Diabetes. 2008;57:1470-81.

43. Xu P, Wang J, Hong F, Wang S, Jin X, Xue T, Jia L, Zhai Y. Melatonin prevents obesity through modulation of gut microbiota in mice. J Pineal Res. 2017;62:e12399.

44. Zhao L. The gut microbiota and obesity: from correlation to causality. Nat Rev Microbiol. 2013;11:639-47.

45. Musso G, Gambino R, Cassader M. Interactions between gut microbiota and host metabolism predisposing to obesity and diabetes. Annu Rev Med. 2011:62:361-80.

46. Tomas J, Reygner J, Mayeur C, Ducroc R, Bouet S, Bridonneau C, Cavin JB, Thomas M, Langella P, Cherbuy C. Early colonizing escherichia coli elicits remodeling of rat colonic epithelium shifting toward a new homeostatic state. ISME J. 2015:9:46-58.

47. van Elburg RM, Fetter WP, Bunkers CM, Heymans HS. Intestinal permeability in relation to birth weight and gestational and postnatal age. Arch Dis Child Fetal Neonatal Ed. 2003;88:F52-5.

48. Guo B, Liu B, Wei H, Cheng KW, Chen F. Extract of the microalga nitzschia laevis prevents high-fat-diet-induced obesity in mice by modulating the composition of gut microbiota. Mol Nutr Food Res. 2019;63:e1800808.

\section{Publisher's Note}

Springer Nature remains neutral with regard to jurisdictional claims in published maps and institutional affiliations.

Ready to submit your research? Choose BMC and benefit from:

- fast, convenient online submission

- thorough peer review by experienced researchers in your field

- rapid publication on acceptance

- support for research data, including large and complex data types

- gold Open Access which fosters wider collaboration and increased citations

- maximum visibility for your research: over $100 \mathrm{M}$ website views per year

At $\mathrm{BMC}$, research is always in progress.

Learn more biomedcentral.com/submissions 